\title{
Metabolic profiling reveals glucose and fructose accumulation in $g c r 1$ knock-out mutant of Arabidopsis
}

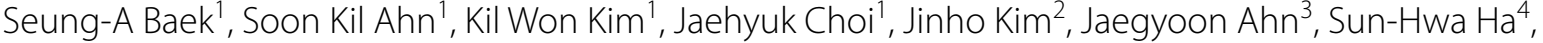 \\ Sang Un Park ${ }^{5^{*}}$ and Jae Kwang Kim ${ }^{1^{*}}$ (1)
}

\begin{abstract}
The ligands and functions of GCR1, the putative G-protein coupled receptor gene of Arabidopsis thaliana, and its role in metabolism are not well studied. Herein, we determined the contents of different pigments, glucosinolates, and lipophilic and hydrophilic compounds in gcr 1 knock-out mutant and wild-type plants to investigate the roles of GCR1. Overall, 68 and 58 metabolites were detected using high performance liquid chromatography, gas chromatographyquadrupole mass spectrometry, and gas chromatography-time-of-flight mass spectrometry in 10-day-old seedlings and 24-day-old shoots of mutant and wild-type plants. The levels of glucose and fructose in the gcr 1 mutant were significantly higher than those in the wild-type at the two developmental stages. The results of partial least squares discriminant analysis and variable importance in the projection showed that glucose and fructose contributed the most to the separation. These results suggest that GCR1 is linked to glucose sensing and affects glycolysis via cyclic AMP.
\end{abstract}

Keywords: Arabidopsis thaliana, GCR1, AtRGS1, G-protein coupled receptor, Metabolic profiling

\section{Introduction}

G-protein coupled receptors (GPCRs) are one of most important membrane-localized receptors. They transduce extracellular signals inside the cells. GPCRs interact with a heterotrimeric complex formed by the coupling of

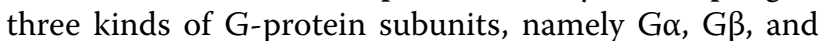
Gy. G-protein signaling is required for the interaction of GPCR with these subunits. When a ligand binds to GPCR, guanosine diphosphate (GDP) of G $\alpha$ is exchanged with guanosine triphosphate (GTP), so that the inactive $G \alpha$ is converted to an active $G \alpha$. The $G \beta \gamma$ dimer is

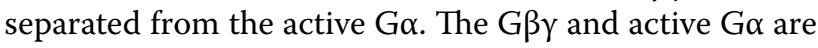
subsequently separated from the GPCR. These subunits then activate downstream signaling in the cell. The active

\footnotetext{
*Correspondence: supark@cnu.ac.kr; kjkpj@inu.ac.kr

${ }^{1}$ Division of Life Sciences, College of Life Sciences and Bioengineering,

Incheon National University, Incheon 22012, Republic of Korea

${ }^{5}$ Department of Crop Science, Chungnam National University, 99,

Daehak-Ro, Yuseong-gu, Daejeon 34134, Republic of Korea

Full list of author information is available at the end of the article
}

G $\alpha$ is deactivated upon hydrolysis of GTP to GDP. The inactive $G \alpha$ is re-complexed with the $G \beta \gamma$ dimer and the heterotrimeric complex combines with the GPCR again. The regulator of G-protein signaling (RGS) protein, a GTPase-activating protein, supports the hydrolysis of GTP and the consequent $\mathrm{G} \alpha$ deactivation [1].

Because of their roles in several metabolic pathways, GPCRs and G-protein signaling have been intensively studied. There are more than 800 types of GPCRs in humans, which regulate adenylyl cyclase, phospholipase C, cyclic GMP phosphodiesterase, and ion channels. Besides humans, GPCRs are conserved in eukaryotes including fungi and plants. In plants, GPCRs have mainly been studied in Arabidopsis and rice $[1,2]$. In Arabidopsis, to date, GCR1 is a putative GPCR gene, which is known to be involved in the processes of DNA synthesis, seed dormancy, and root and flower development [3-7]. Also, there are five kinds of G-protein subunits (one $\alpha$ subunit, one $\beta$ subunit, and three $\gamma$ subunits) in Arabidopsis. These subunits are involved in different 
signaling processes, such as hormone and glucose signaling, and in stress response and formation of organ structures. Furthermore, plant-specific G-proteins have three extra-large $\mathrm{G} \alpha$ proteins (XLGs). The XLGs are involved in growth, development, and defending systems [8-10]. The results of transcriptome analysis confirmed that GCR1 affects the response of plants to biotic and abiotic stress, hormones, secondary metabolism, and phosphate starvation [11, 12]. However, the accurate roles of GCR1 remain unknown. Because GCR1 is known to be involved in germination, development, flowering, ripening of fruits, senescence, and in response to various stresses, either directly or indirectly, it could be an important gene for increasing the yield of plants.

As of date, only genomics and transcriptomics studies have been conducted in relation to GCR1, and metabolomics has not been employed. Like genomics, transcriptomics, and proteomics, metabolomics is an important "-omics" technique. It has been used in the analysis of metabolic pathways, identification of biomarkers, and for deciphering metabolic mechanisms. Metabolic profiling is the analysis of quantitative and qualitative changes in the metabolites present in samples using analytical techniques, such as liquid and gas chromatography.

In the present study, we performed metabolic profiling to investigate the metabolic changes in a gcrl knock-out mutant of Arabidopsis with respect to the metabolites present in the wild-type (Col-0) plants. We used highperformance liquid chromatography (HPLC), gas chromatography-quadrupole mass spectrometry (GC-qMS), and gas chromatography-time-of-flight mass spectrometry (GC-TOFMS), to detect different metabolites, such as carotenoids, policosanols, tocopherols, phytosterols, amino acids, organic acids, inorganic acids, sugars, and sugar alcohols, in two developmental stages of Arabidopsis. Because previous studies have shown that GCR1 regulates the timings of germination and flowering [1], 10-day-old seedlings and 24-day-old shoot samples were used to represent the cotyledonary stage and the stage just before flowering, respectively. We believe that data regarding the differences in the metabolites present in gcr 1 knock-out mutant and wild-type Arabidopsis plants, would provide fundamental information for understanding the role of GCR1 and the pathway in which it is involved that could be instrumental in formulating strategies for increasing the yield and levels of useful nutrients in plants.

\section{Materials and methods}

\section{Plant materials and growth conditions}

The mutant seeds with T-DNA insertion (Stock name CS6539) were obtained from Arabidopsis Biological Resource Center (ABRC, Ohio State University,
Columbus, OH, USA). Arabidopsis thaliana wild-type Columbia-0 (Col-0) was used as the control. The seeds were sterilized by dipping in $1 \%$ sodium hypochlorite containing $0.01 \%$ Tween 20 for $7 \mathrm{~min}$, and in $70 \%$ ethanol for $5 \mathrm{~min}$; this was followed by rinsing with sterile water for five times. After vernalization at $4{ }^{\circ} \mathrm{C}$ for 3 days in dark, the seeds were sown on sterile water. The seeds were grown in a growth chamber at $25 \pm 1{ }^{\circ} \mathrm{C}$ under a light intensity of approximately $4000 \mathrm{~lx}$ and a $16 \mathrm{~h} \mathrm{light} / 8 \mathrm{~h}$ dark photoperiod for 10 days. The humidity was $50 \% \pm 5 \%$. For the experiments in which 24 -dayold plants were required, the seeds were sown directly in plastic pots containing $400 \mathrm{~g}$ of autoclaved commercial soil (Singsingnara; Seoul Bio Co., Eumseong, Korea). The pots were placed in a dark chamber at $4{ }^{\circ} \mathrm{C}$ for vernalization. After 3 days, the pots were transferred to the growth chamber maintained under the conditions mentioned above and watered using sub-irrigation. The harvested samples were homogenized in liquid nitrogen and kept at $-80^{\circ} \mathrm{C}$ until the gene expression and metabolites analyses.

\section{Isolation of $g c r 1$ knock-out mutant and gene expression analysis}

For isolation of the $g c r 1$ knock-out mutant, DNA was extracted from 10-day-old whole seedlings and shoots of 24-day-old plants using the modified Cone's method [13]. The amplification was done using TaKaRa Ex Taq (Takara Bio, Shiga, Japan) in Veriti 96 Well Thermal Cycler (Applied Biosystems, Foster City, CA, USA). The PCR mixture contained $0.5 \mu \mathrm{g}$ of template, 10X Ex Taq buffer, dNTP mixture ( $2.5 \mathrm{mM}$ of each dNTP), $0.2 \mu \mathrm{M}$ of each primer, and $0.2 \mu \mathrm{L}$ of TaKaRa Ex Taq (5 units) in a total volume of $20 \mu \mathrm{L}$. The PCR conditions were as follows: $95{ }^{\circ} \mathrm{C}$ for $4 \mathrm{~min}$, followed by 35 cycles of $95{ }^{\circ} \mathrm{C}$ for $45 \mathrm{~s}, 60{ }^{\circ} \mathrm{C}$ for $45 \mathrm{~s}$, and $72{ }^{\circ} \mathrm{C}$ for $90 \mathrm{~s}$, and a final extension step at $72{ }^{\circ} \mathrm{C}$ for $10 \mathrm{~min}$.

Total RNA was isolated from 10-day-old whole seedlings and 24-day-old shoots using RNeasy Plant Mini kit (Qiagen, Hilden, Germany). After treatment with RQ1 RNase-free DNase (Promega, Madison, WI, USA), the first strand cDNA was synthesized with an oligo $(\mathrm{dT})_{15}$ primer (Promega) using AccuPower RT PreMix (Bioneer, Daejeon, Korea). The loss of GCR1 transcript was checked using reverse transcriptase PCR (RT-PCR). The reaction mixture $(20 \mu \mathrm{L})$ contained the template $(0.5-$ $2.5 \mu \mathrm{g}), 0.2 \mu \mathrm{M}$ of each primer, and EmeraldAmp PCR master Mix (Takara). The amplification conditions were as follows: $95^{\circ} \mathrm{C}$ for $3 \mathrm{~min}$, followed by 35 cycles of $95^{\circ} \mathrm{C}$ for $15 \mathrm{~s}, 55^{\circ} \mathrm{C}$ for $15 \mathrm{~s}$, and $72{ }^{\circ} \mathrm{C}$ for $1 \mathrm{~min}$, and a final extension step at $72{ }^{\circ} \mathrm{C}$ for $2 \mathrm{~min}$.

The quantitative real-time PCR (qRT-PCR) was carried out on GFX Connect Real-Time System (Bio-Rad, 
Hercules, CA, USA). The reaction mixture contained $0.5 \mu \mathrm{g}$ of $\mathrm{cDNA}, 0.6 \mu \mathrm{L}(10 \mathrm{pm} / \mu \mathrm{L})$ of each primer, and $10 \mu \mathrm{L}$ of iQ SYBR Green Supermix (Bio-Rad) in a total volume of $20 \mu \mathrm{L}$. The amplification conditions were as follows: $95^{\circ} \mathrm{C}$ for $3 \mathrm{~min}$, followed by 40 cycles of $10 \mathrm{~s}$ at $95{ }^{\circ} \mathrm{C}$ and $30 \mathrm{~s}$ at $60{ }^{\circ} \mathrm{C}$, and $95^{\circ} \mathrm{C}$ for $10 \mathrm{~s}$; this was followed by increasing the temperature from 65 to $95^{\circ} \mathrm{C}$ to check for the amplification specificity. The expression of GCR1 relative to AtEF1 $\alpha$ was determined using the $2^{-}$ ${ }^{\mathrm{ddCt}}$ method [14]. The primers used in this study are listed in Additional file 1: Table S1.

\section{Extraction of carotenoids and HPLC analysis}

The methods for extraction and analysis of carotenoids were as described by Park et al. [15]. Qualitative and quantitative analyses of carotenoids were done on freshly powdered (300 mg) samples using calibration curves made for the standard compounds.

\section{Extraction of chlorophylls and analysis}

Total chlorophylls were extracted from 10 to $30 \mathrm{mg}$ of fresh samples using $100 \%$ methanol at $70{ }^{\circ} \mathrm{C}$ for $30 \mathrm{~min}$ with Thermomixer Comport (Eppendorf AG, Hamburg, Germany) at $500 \mathrm{rpm}$ speed. The sample was centrifuged at $4{ }^{\circ} \mathrm{C}$ and $3000 \mathrm{rpm}$ for $10 \mathrm{~min}$, and the absorbance of the supernatant was measured at 666 and $653 \mathrm{~nm}$ to calculate the chlorophyll content using the formula mentioned by Wellburn [16].

\section{Extraction of lipophilic compounds and GC-qMS analysis} Freshly powdered samples (300 mg) were used to extract policosanols, tocopherols, and phytosterols. The extraction, and qualitative and quantitative analyses were conducted using previously described methods [17].

\section{Extraction of desulfoglucosinolates and HPLC analysis}

Extraction of desulfoglucosinolates from $100 \mathrm{mg}$ of sample and identification of peaks was done using the procedure described by Baek et al. [18]. The content of glucosinolate was calculated using the response factor of each compound relative to that of sinigrin [19].

\section{Extraction of hydrophilic metabolites and GC-TOFMS analysis}

Fresh samples $(100 \mathrm{mg})$ were used for analysis of hydrophilic metabolites. The qualitative analysis was performed by comparison with the respective standards, possessed in an in-house library [20]. The quantitative estimation was based on peak area ratios relative to the IS peak area.

\section{Statistical analysis}

All the experiments were carried out in triplicates. The data for metabolites were normalized (unit variance scaling) and analyzed using SIMCA (version 14.1, Umetrics, Umea, Sweden). The PCA and PLS-DA were used for visualization of data for multiple metabolites from $A$. thaliana. The results of Student's $t$-test were obtained from MetaboAnalyst 4.0 (http://www.metab oanalyst.ca). Pearson's correlation analysis, hierarchical clustering analysis (HCA), and heat map visualization were also conducted using MetaboAnalyst 4.0.

\section{Results and discussion}

\section{Confirmation of GCR1 expression}

Before metabolite analysis, PCR, RT-PCR, and qRTPCR were performed using specific primers to check for the presence and expression of GCR1 transcript in 10- and 24-day-old samples. The T-DNA insert was located in the sixth exon of the at 1948270 sequence, which is a putative GPCR gene in Arabidopsis [5, 21]. The PCR results showed that the T-DNA fragment was inserted in the gcr 1 mutants. The RT-PCR and qRTPCR showed the absence of GCR1 transcript in the mutants, which was present in the wild-type (Col-0) plants (Fig. 1).

\section{Metabolite profiling}

This study describes the first assessment of the primary and secondary metabolites in gcr 1 knock-out mutants and their comparison with the metabolites present in Col-0 using HPLC, GC-qMS, and GC-TOFMS. A total of nine different types of carotenoids were detected by HPLC (Additional file 1: Figs. S1 and S2). Among these, two types of phytoene isomers, $15-Z$-phytoene and all- $E$-phytoene, were identified in the 10-day-old seedlings, but not in the 24-day-old shoot samples. Policosanols, tocopherols, and phytosterols were identified by GC-qMS. A total of 17 different types of lipophilic compounds (9 policosanols, 3 tocopherols, and 5 phytosterols) were detected in the seedling samples. Sixteen different types of lipophilic compounds, excluding C23, were detected in the shoot samples. Glucosinolates are known as defensive compounds against herbivores and insects and are primarily present in Brassicaceae [22]. Eight different types of glucosinolates, including 5 aliphatic and 3 indolic glucosinolates, were detected in all the samples using HPLC (Additional file 1: Figs. S3 and S4). Using GC-TOFMS, 17 amino acids, 9 organic acids, 4 sugars and 1 sugar alcohol were identified in the 10-day-old seeding samples. Six amino acids, 10 organic acids, 7 sugars, and 1 sugar alcohol were identified in the 


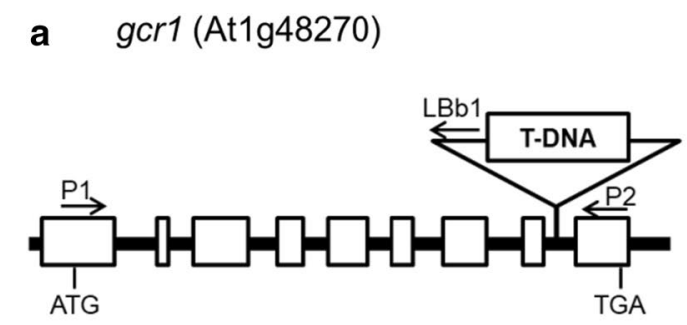

C

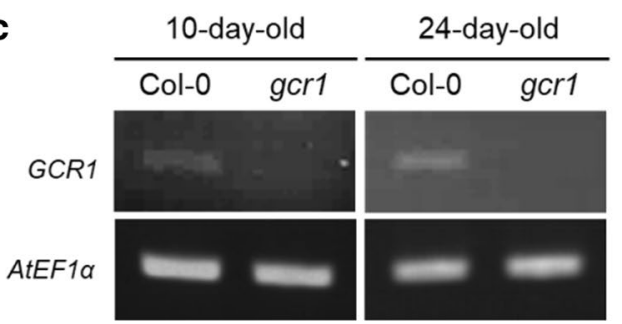

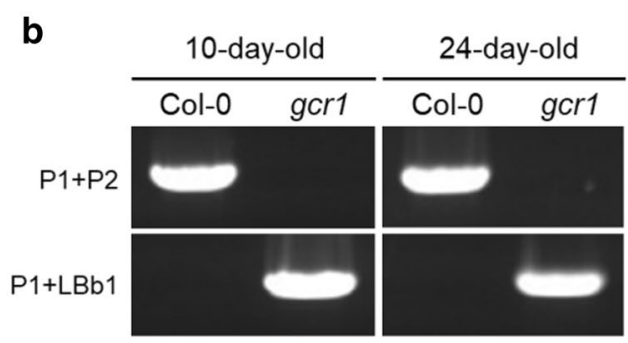

d

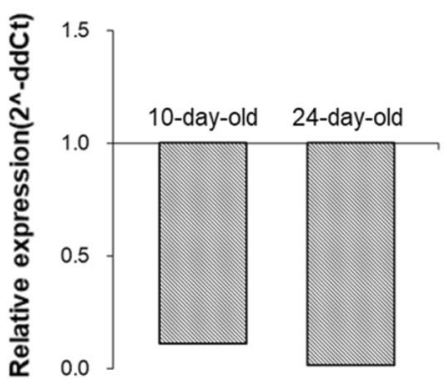

Fig. 1 T-DNA insertion mutation in the gcrl gene in Arabidopsis thaliana and its expression pattern as analyzed by genotyping, and RT-PCR and qRT-PCR (a) diagram showing the position of T-DNA insertion based on the flanking sequences. Black line and white boxes represent the introns and exons, respectively. $\mathbf{b}$ Genotyping PCR of the T-DNA insertion knock-out line (gcr1). The primers used were as follows: P1, 5'-UTR region primer; P2, 3'-UTR region primer; LBb1, T-DNA left border primer. $\mathbf{c}$ RT-PCR analysis of the GCR1 transcript. $\mathbf{d}$ qRT-PCR validation of the mutant. The relative expression of the GCR1 transcript was calculated by the delta-delta $\mathrm{Ct}(\mathrm{ddCt})$ method and was converted to relative expression ratio $\left(2^{-\mathrm{dd} C \mathrm{t}}\right)$. The expression of AtEF1a was used as the control. The primers used for the analyses are listed as in Additional file 1: Table S1

24-day-old shoot samples (Additional file 1: Figs. S5 and S6).

\section{PCA, PLS-DA, and HCA}

The principal component analysis (PCA) and PLSDA were performed to visualize the multivariate data obtained from the gcrl mutant and wild-type samples. The aim of PCA and PLS-DA is to identify class differences in a multivariate dataset [23]. The PCA analysis revealed a clear separation between the data obtained for gcr 1 mutant and control samples of seedlings and shoot parts (Additional file 1: Figs. S7 and S8). To predict the roles of GCR1, it was necessary to focus on the most contributed metabolites for separation. We, therefore, performed PLS-DA, which makes it possible to accomplish a rotation of the projection to give latent variables that focus on class separation. This method explicitly considers the class membership of observations. Thus, PLS-DA develops a model that separates classes of observations on the basis of their original X-variables, which were the data obtained for $g c r 1$ mutant and wild-type plants in the present case [24].

In PLS-DA performed for 68 metabolites detected in the 10-day-old seedling samples, the gcr 1 mutant and wild-type were separated into two parts by $\mathrm{x}$-axis (PLS 1) (Fig. 2). The loading plot indicated that the significant metabolites for separation were fructose, glucose, and inositol, and the eigenvectors of PLS 1 were 0.1626 , 0.1614 , and 0.1611 , respectively. The positive eigenvector means that the $g c r 1$ mutant has relatively higher levels of these metabolites. On the other hand, $\beta$-carotene, $13 Z$ - $\beta$-carotene, and amino acids, such as glutamic acid, asparagine, and glutamine were at higher levels in the wild-type plants (eigenvectors were $-0.1620,-0.1613$ $-0.1623,-0.1602$, and -0.1600 , respectively). Most of the primary and secondary metabolites had negative eigenvectors, which indicated that the wild-type plants had relatively higher levels of amino acids, organic acids, carotenoids, chlorophylls, policosanols, tocopherols, phytosterols, and glucosinolates compared to their levels in the gcr 1 mutant. In the 24-day-old shoot samples, the result of PLS-DA using 58 metabolites also showed the separation of data obtained for the mutant and wildtype plants (Fig. 3). Glucose, fructose, and raffinose were verified as the significant metabolites contributing to the separation in the loading plot. They had positive eigenvectors of PLS 1 (0.2389, 0.2381, and 0.1968 , respectively), indicating that these metabolites were present at higher levels in the gcrl mutant. However, the levels of photosynthesis pigments, glyceric acid, TCA cycle intermediates (pyruvic acid and fumaric acid), and sucrose were relatively higher in the control plant. 


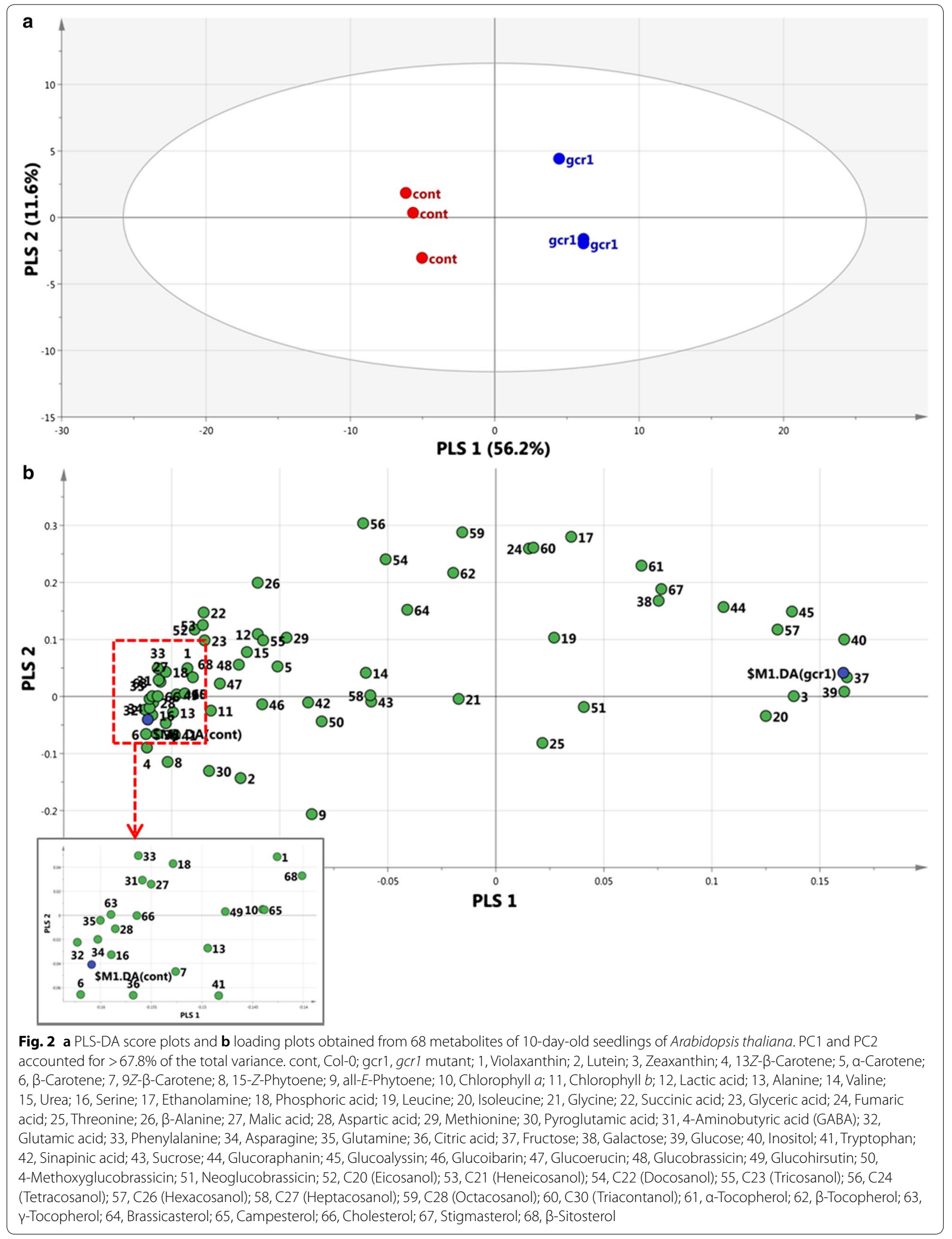




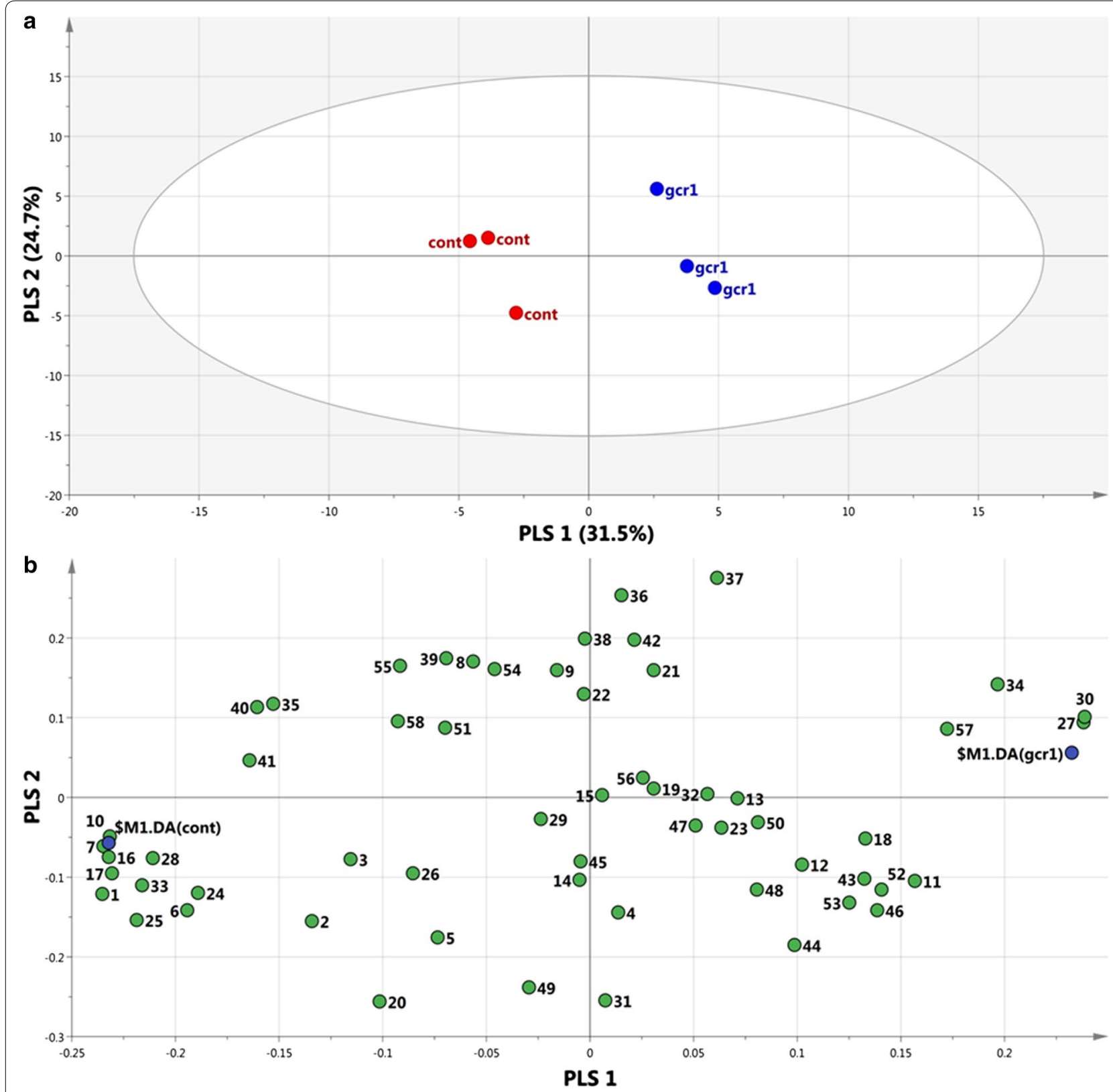

Fig. 3 a Score plots of partial least squares discriminant analysis (PLS-DA) and $\mathbf{b}$ loading plots obtained from metabolite data of 24-day-old shoots. PC1 and PC2 accounted for $>56.2 \%$ of the total variance. cont, Col-0; gcr1, gcr1 mutant; 1, Violaxanthin; 2, Lutein; 3, Zeaxanthin; 4, 13Z- $\beta$-Carotene; 5, a-Carotene; 6, $\beta$-Carotene; 7, 9Z- $\beta$-Carotene; 8, Chlorophyll $a ; 9$, Chlorophyll $b ; 10$, Pyruvic acid; 11, Lactic acid; 12, Serine; 13, Phosphoric acid; 14 , Glycine; 15, Succinic acid; 16, Glyceric acid; 17, Fumaric acid; 18, $\beta$-Alanine; 19, Malic acid; 20, Pyroglutamic acid; 21, 4-Aminobutyric acid (GABA); 22 , Threonic acid; 23, Glutamic acid; 24, Xylose; 25, Shikimic acid; 26, Citric acid; 27, Fructose; 28, Mannose; 29, Galactose; 30, Glucose; 31, Inositol; 32, Sinapinic acid; 33, Sucrose; 34, Raffinose; 35, Glucoraphanin; 36, Glucoalyssin; 37, Glucoibarin; 38, Glucoerucin; 39, Glucobrassicin; 40, Glucohirsutin; 41, 4-Methoxyglucobrassicin; 42, Neoglucobrassicin; 43, C20 (Eeicosanol); 44, C21 (Heneicosanol); 45, C22 (Docosanol); 46, C24 (Tetracosanol); 47, C26 (Hexacosanol); 48, C27 (Heptacosanol); 49, C28 (Octacosanol); 50, C30 (Triacontanol); 51, a-Tocopherol; 52, ß-Tocopherol; 53, Y-Tocopherol; 54, Brassicasterol; 55, Campesterol; 56, Cholesterol; 57, Stigmasterol; 58, $\beta$-Sitosterol

Variable importance in the projection (VIP) is a weighted sum of the squared PLS weights. The metabolites with a larger VIP (larger than 1.0) are more influential for the model [24]. In the seedling sample set, 37 metabolites, including fructose, glucose, $\beta$-carotene, and glutamic acid, had a VIP value higher than 1.0 (Fig. 4). In the shoot samples, 22 types of metabolites including sugars, carotenoids, and organic acids, 

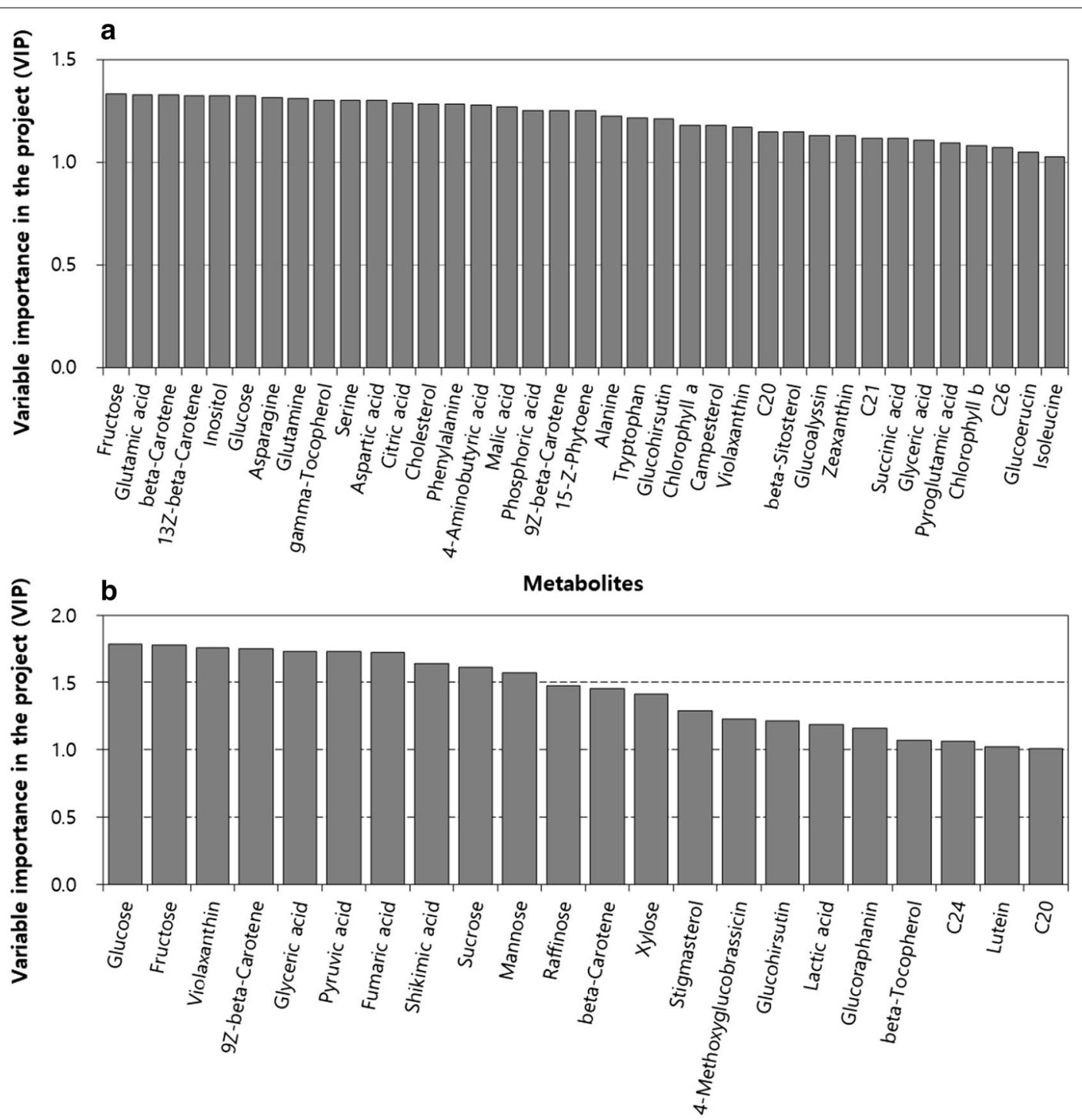

Metabolites

Fig. 4 Influence of variables used to create a distinction model for gcr1 mutant and wild (Col-0) plants for a 10-day-old seedlings and b 24-day-old shoots. Metabolites with higher variable importance in the projection (VIP) values are more influential for the sample separation. Metabolites with VIP values > 1.0 are shown. C20, Eicosanol; C21, Heneicosanol; C24, Tetracosanol; C26, Hexacosanol

were found to be significant compounds for separation (VIP value >1.0). Glucose had the highest VIP value (1.7829), and was followed by fructose, violaxanthin, $9 Z$ - $\beta$-carotene, glyceric acid, pyruvic acid, and fumaric acid.
To decipher the detailed relationship among the metabolites in Arabidopsis, Pearson's correlation analysis and HCA were performed using MetaboAnalyst 4.0. The correlation coefficient $(r)$ was in the range $-1<r<1$. A value of $r>0$ indicates a positive association and $r<0$

(See figure on next page.)

Fig. 5 Results of correlation matrix and clustering analysis obtained from the data of 68 metabolites present in $\mathbf{a}$ 10-day-old seedlings and $\mathbf{b}$ 24-day-old shoots of gcr1 mutant and wild-type (Col-0) Arabidopsis thaliana plants. Each square indicates the Pearson's correlation coefficient of a pair of compounds, and the value for the correlation coefficient is represented by the intensity of the blue or red color, as indicated on the color scale. Hierarchical clusters are represented by a cluster tree. C20, Eicosanol; C21, Heneicosanol; C22, Docosanol; C23, Tricosanol; C24, Tetracosanol; C26, Hexacosanol; C27, Heptacosanol; C28, Octacosanol; C30, Triacontanol 


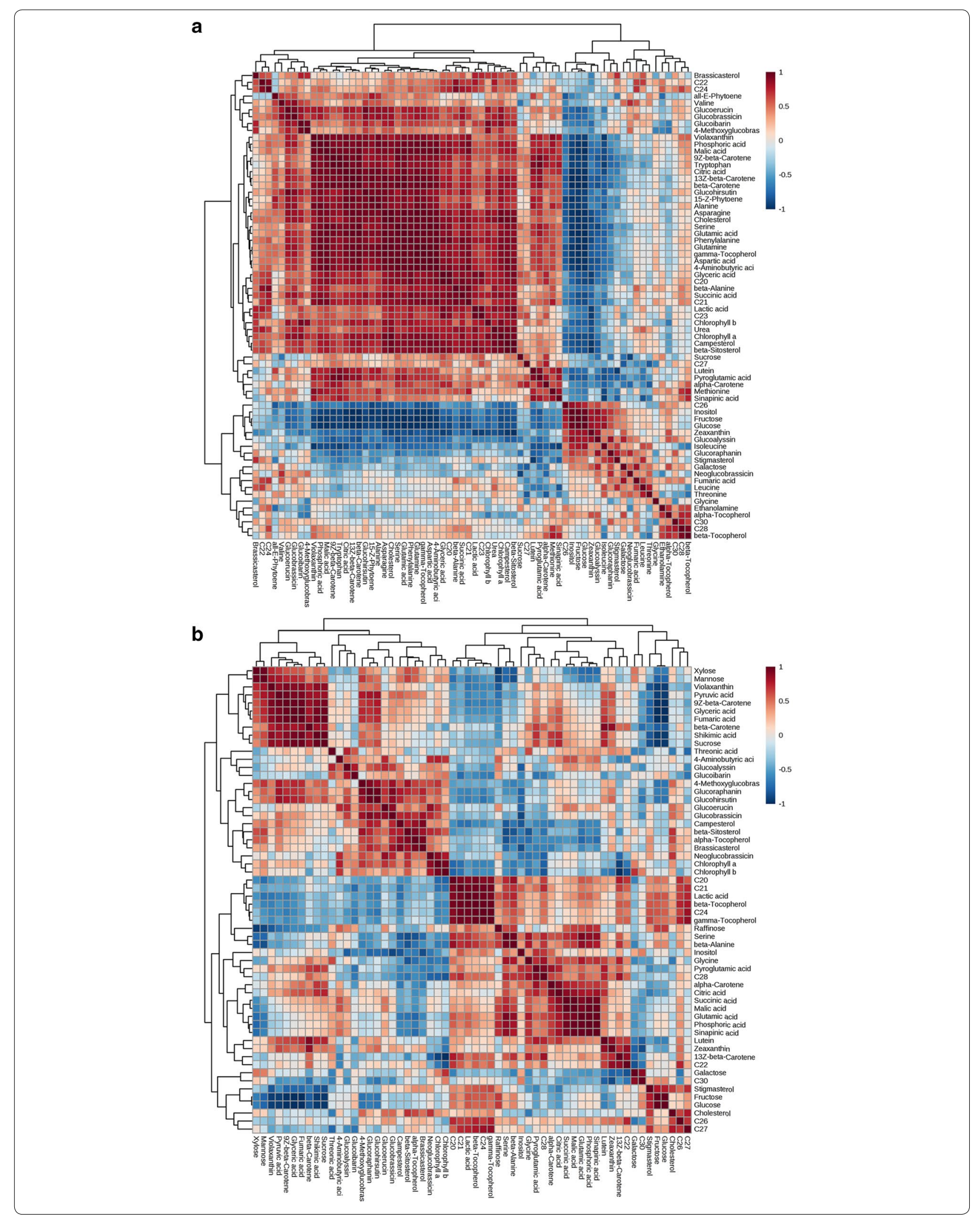


indicates a negative association between two metabolites. In the seedling samples, the HCA provided two major metabolite clusters (Fig. 5). In one group, the carotenoids, except zeaxanthin and chlorophylls, were clustered together. This group included malic acid, citric acid, and succinic acid. Another group included inositol, fructose, glucose, and galactose. This clustering showed the same pattern as observed in the loading plot of PLSDA. Glucose, fructose, and inositol showed positive correlation with one another, but had a negative correlation with the TCA cycle intermediates. The correlation coefficient of glucose and citric acid was -0.9640 , and that of glucose and succinic acid was -0.8692 . In the shoot samples, instead of inositol, raffinose showed a positive correlation coefficient with glucose $(r=0.6790)$ and fructose $(r=0.6672)$. Glucose showed negative correlation coefficients with pyruvic acid, citric acid, succinic acid, and malic acid $(r=-0.9450,-0.5189,-0.1728$, and -0.0722 , respectively).

\section{Differences in the metabolite contents between gcr1 mutant and wild-type plants}

The results of PLS-DA and HCA revealed that glucose, fructose, and $\beta$-carotene were the metabolites that differed the most between the seedling and shoot samples of gcrl mutant and wild-type plants. On the basis of these results, the actual quantitative values were compared. In the seedling samples, the total carotenoid content in the $\mathrm{gcr} 1$ mutant was $19.70 \pm 2.42 \mu \mathrm{g} / \mathrm{g}$ and that in the wild-type was $28.36 \pm 2.16 \mu \mathrm{g} / \mathrm{g}$ of fresh weight (FW). The total chlorophyll content in the $g c r 1$ mutant $(78.28 \pm 7.73 \mu \mathrm{g} / \mathrm{g}$ of FW) was lower than in the wild-type $(99.71 \pm 6.46 \mu \mathrm{g} / \mathrm{g}$ of FW) (Additional file 1: Table S2). There were no noticeable differences in the total content of policosanols, tocopherols, phytosterols, and glucosinolates between the mutant and wild-type plants (Additional file 1: Tables S3 and S4). Among the hydrophilic compounds, the contents of most of the metabolites were higher in the wild-type samples, but fructose, glucose, and inositol were the exceptions and had relatively higher levels in the mutant samples (Additional file 1: Table S5). The differences in the detected metabolites are presented as heat maps in the schematic representation of metabolic pathways (Fig. 6).

To identify the differences in the content of each metabolite between the mutant and wild samples, Student's $t$-test was conducted using MetaboAnalyst 4.0. Thirty-three metabolites showed significant differences ( $p$-value $<0.05)$ between the mutant and wild-type samples (Additional file 1: Fig. S9). Especially, 10 compounds, including fructose, glutamic acid, $\beta$-carotene, glucose, and inositol showed highly significant differences ( $p$-value $<0.001)$. In the shoot samples, there were no striking differences in the total content of pigments, lipophilic compounds, and glucosinolates between the $\mathrm{gcr} 1$ mutant and wild-type samples (Additional file 1: Tables S3 and S4). However, as in the seedling samples, the contents of fructose and glucose were relatively higher in the mutant shoot samples (Additional file 1: Table S5). Based on the results of the $t$-test, there were 10 metabolites that had $p$-value lower than 0.05 (Additional file 1: Fig. S10). Only glucose and fructose showed highly significant differences ( $p$-value $<0.001)$.

In this study, the quantitative and qualitative analysis of primary and secondary metabolites in the gcr 1 knock-out mutant and wild-type (Col-0) plants were conducted at two different developmental stages. The statistical analysis showed that there were certain differences in the levels of monosaccharides between the $g c r 1$ mutant and wildtype plants. In other words, glucose and fructose were accumulated in the $g c r 1$ mutants of 10-day-old seedlings and 24-day-old shoot. The gcr1 mutant has low sensitivity to gibberellin and brassinosteroid during germination [5], but has high sensitivity to ABA [7]. These results could be explained, if the accumulated glucose and fructose influence the hormone signaling and responses. ABA synthesis is increased by glucose via the GIN2 (AtHXK1) gene. The GIN2 (AtHXK1) gene also affects the auxin, cytokinin, and ethylene signaling pathways [25]. The DELLA pathway connects gibberellin to the response of other hormones [26]. As a stress hormone, ABA induces seed dormancy, stomatal closure, root growth, and senescence. Gibberellin and ethylene have effects contrary to that of ABA. Gibberellin promotes flowering, germination, and cotyledon greening, which is dependent on brassinosteroids. The increased level of ABA reduces the effects of gibberellin and brassinosteroids, thereby, decreasing the percentage of germination. The high sensitivity of the $g c r 1$ mutant to ABA was supposed to be a synergistic effect of the increased ABA content [7]. In the seedling sample, the level of total pigments was lower in the gcr 1 mutant $(97.98 \pm 10.15 \mu \mathrm{g} / \mathrm{g}$ of FW) than in the wildtype $(128.07 \pm 8.62 \mu \mathrm{g} / \mathrm{g}$ of FW). In addition, the results of PLS-DA and VIP value indicate that $\beta$-carotene, the predominant carotenoid, was one of the significant compounds for separation. The increase in carotenoids was because of the ABA response, and it prevents the greening of cotyledons [27]. Therefore, high glucose level in the mutant plants ultimately affected ABA and the response of other hormones via GIN2 and DELLA.

The accumulation of glucose and fructose in the gcr 1 mutant could be explained by comparing the results of transcriptomics study. The flavonoid biosynthesis pathway genes were up-regulated (flavanone 3beta-hydroxylase; at5g20550, at2g36690) and down-regulated (flavonol synthase; at3g49620) in the GCR1 mutant (gcr1-5) [12]. 


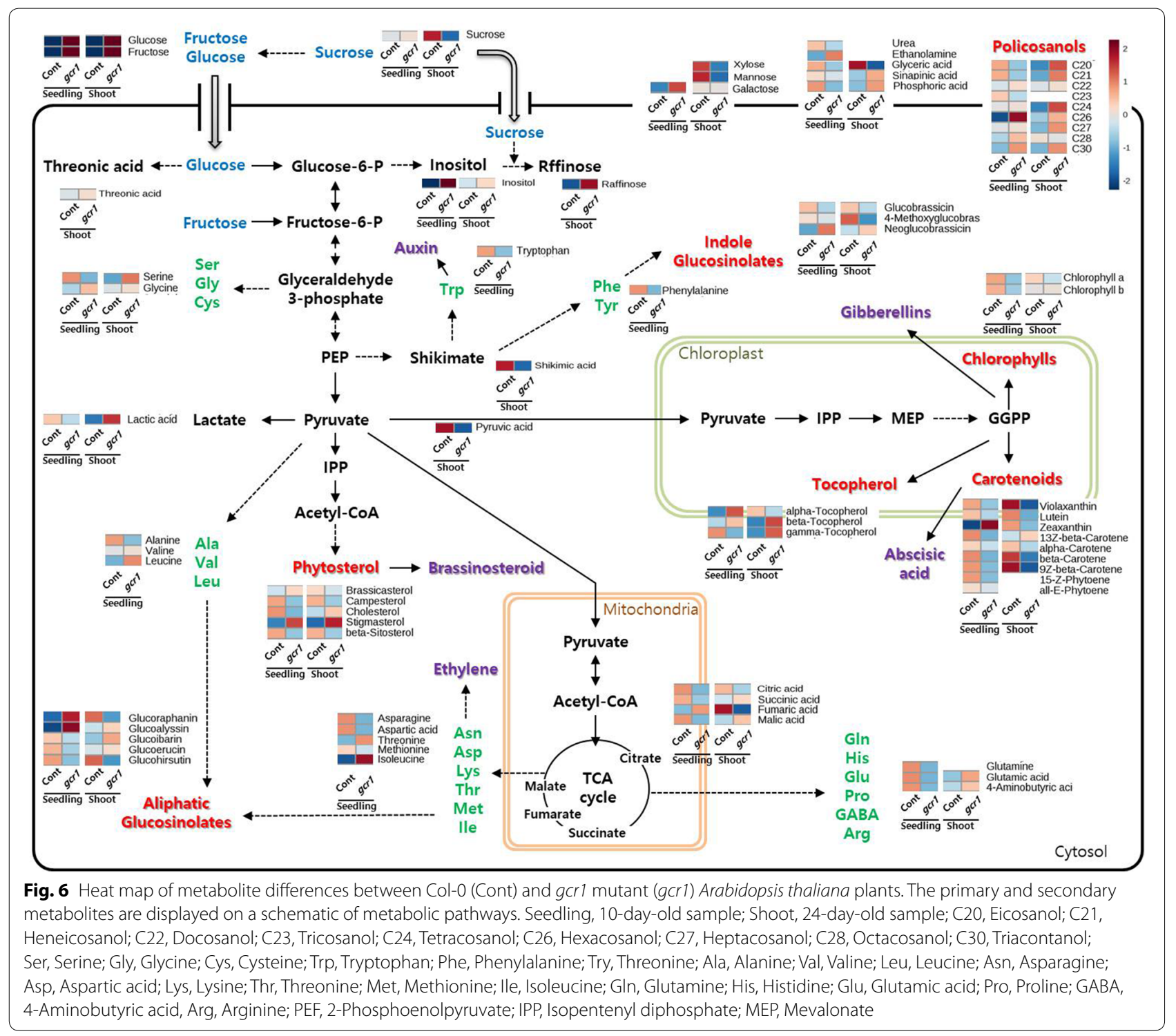

The flavonols (quercetin, kaepferol) are synthesized from dihydroflavonols by flavonol synthase. The lower level of this enzyme induced lower levels of flavonols, which ultimately inhibited the formation of flavonoid glucosides, and then glucose could be accumulated in the mutant.

Apone et al. [3] reported that phosphatidylinositolspecific phospholipase C (PI-PLC) activity and inositol 1,4,5-triphosphate $\left(\mathrm{IP}_{3}\right)$ were increased in tobacco cells overexpressing Arabidopsis GCR1 and GPA1 genes. In mammalian cells, $\mathrm{IP}_{3}$ and diacylglycerol (DAG) are produced from phosphatidylinositol 4,5-bisphosphate $\left(\mathrm{PIP}_{2}\right)$ by hydrolysis of phospholipase $\mathrm{C}$ (PLC). $\mathrm{IP}_{3}$ is hydrolyzed by gradual steps to inositol and inorganic phosphate $(\mathrm{Pi})$. The free inositol is recycled into $\mathrm{PIP}_{2}$ [28]. If this process existed in plants, the high level of inositol in $\mathrm{gcr} 1$ mutant could be explained. The decreased activity of PLC affected the conversion of inositol and other intermediates into PIP ${ }_{2}$. In cytosol, infused $\mathrm{IP}_{3}$ induces the release of $\mathrm{Ca}^{2+}$ from intracellular stores [29]. $\mathrm{Ca}^{2+}$ is important as a secondary messenger in plants in response to various stimuli, including salinity, heat, cold, pathogens, hormones, and wounding [30]. Therefore, the different phenotypes of GCR1 mutant could appear as an effect of the change in cytosolic $\mathrm{Ca}^{2+}$ content via phospholipase (Additional file 1: Fig. S11).

In addition, another hypothesis could be proposed that GCR1 regulates the cyclic adenosine monophosphate (cAMP) signaling pathway. When an unknown ligand binds to GCR1, cAMP is induced and it regulates the down-stream signaling. Alqurash et al. [31] detected that 
fructose bisphosphate aldolase was accumulated in $A$. thaliana cell suspension culture treated with cAMP. This enzyme converts fructose 1,6-bisphosphate to glyceraldehyde 3-phosphate in the glycolysis pathway. In the gcrl mutant, the level of cAMP was decreased, which led to low levels of bisphosphate aldolase. Eventually, fructose 1,6-bisphosphate and the up-stream metabolites (glucose, fructose, raffinose, and inositol) were accumulated. In addition, the down-stream signaling of glyceraldehyde 3-phosphate was also disturbed. It could influence the development, response to a stimulus, biosynthesis of metabolites, and other physiological actions.

The relationship of GPCR and cAMP signaling has been detected in humans and yeast [32, 33]. In addition, it is known that glucose activates the cAMP pathway via a GPCR in yeast. In A. thaliana, glucose sensing and its regulation of G-protein signaling through AtRGS1 has previously been reported [34]. Glucose and fructose sustain the activation of the Ga subunit through endocytosis of plant 7TM-RGS. In this study, gcr1 mutant could not recognize glucose; it inhibited the endocytosis of 7TMRGS so that hydrolysis of GTP from the Go subunit was induced. Consequently, G-protein signaling was inactivated and its down-stream signaling pathways, such as cAMP signaling, were interrupted. Therefore, the present results suggest the potential role of GCR1 in inducing the cAMP signaling through sensing of glucose (Additional file 1: Fig. S11).

Our results provide evidence for the role of GCR1 and reveal the pathways in which it could be involved. Furthermore, our study shows that metabolic profiling is a helpful method to investigate the unknown functions of a gene. The future work would focus on analyzing the relative gene expression and measuring the enzyme activity under the same conditions that would support our hypothesis. The results and methods used in this study can be applied to investigate the functions of GPCRs in other species of Brassicaceae, as well as in rice, maize, and other major crops.

\section{Additional file}

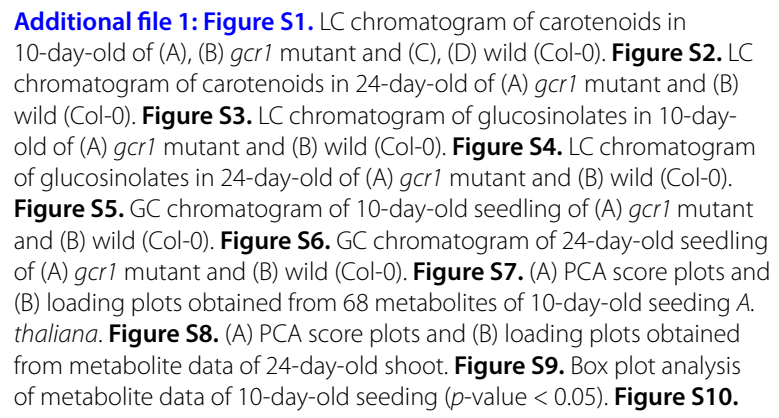

Additional file 1: Figure S1. LC chromatogram of carotenoids in 10-day-old of (A), (B) gcr1 mutant and (C), (D) wild (Col-0). Figure S2. LC chromatogram of carotenoids in 24-day-old of (A) gcr1 mutant and (B) wild (Col-0). Figure S3. LC chromatogram of glucosinolates in 10-dayold of (A) gcrl mutant and (B) wild (Col-0). Figure S4. LC chromatogram of glucosinolates in 24-day-old of (A) gcr 1 mutant and (B) wild (Col-0). Figure S5. GC chromatogram of 10-day-old seedling of (A) gcr1 mutant and (B) wild (Col-0). Figure S6. GC chromatogram of 24-day-old seedling of (A) gcr1 mutant and (B) wild (Col-0). Figure S7. (A) PCA score plots and (B) loading plots obtained from 68 metabolites of 10-day-old seeding $A$. thaliana. Figure S8. (A) PCA score plots and (B) loading plots obtained from metabolite data of 24-day-old shoot. Figure S9. Box plot analysis of metabolite data of 10-day-old seeding ( $p$-value $<0.05$ ). Figure S10.

Box plot analysis of metabolite data of 24-day-old shoot ( $p$-value $<0.05$ ). Figure S11. The hypothetial model of glucose induced signaling through GCR1. Table S1. The sequences of the primers used in this study. Table S2. Composition and abundance ( $\mu \mathrm{g} / \mathrm{g}$ of FW) of pigments in wildtype (Col-0) and gcr 1 knock-out mutant ( $g$ cr 1 ) plants. Table S3. Composition and abundance $(\mu \mathrm{g} / \mathrm{g}$ of FW) of policosanols, tocopherols, and phytosterols in the wild-type (Col-0) and gcr 1 knock-out mutant (gcr1) plants. Table S4. Composition and abundance ( $\mu \mathrm{g} / \mathrm{g}$ of FW) of glucosinolates in the wild-type (Col-0) and gcr 1 knock-out mutant (gcr1) plants. Table S5.

Composition and abundance (ratio/g of FW) of hydrophilic compounds in the wild-type (Col-0) and gcr knock-out mutant (gcri) plants.

\section{Authors' contributions}

SAB analyzed the data and wrote the paper. SKA and SHH provided advice of experimental design. KWK, JC, JK and JA conceived the concept and designed the experiments. SUP and JKK revised and edited the manuscript and supervised the work. All authors read and approved the final manuscript.

\section{Author details}

1 Division of Life Sciences, College of Life Sciences and Bioengineering, Incheon National University, Incheon 22012, Republic of Korea. ${ }^{2}$ Division of Chemistry, College of Natural Science, Incheon National University, Incheon 22012, Republic of Korea. ${ }^{3}$ Division of Computer Science and Engineering, College of Information Technology, Incheon National University, Incheon 22012, Republic of Korea. ${ }^{4}$ Department of Genetic Engineering and Graduate School of Biotechnology, Kyung Hee University, Yongin 17104, Republic of Korea. ${ }^{5}$ Department of Crop Science, Chungnam National University, 99, Daehak-Ro, Yuseong-gu, Daejeon 34134, Republic of Korea.

\section{Acknowledgements}

This work was supported by 2018 research program funded by the Institute of Convergence Science and Technology, Incheon National University, Republic of Korea.

\section{Competing interests}

The authors declare that they have no competing interests.

\section{Publisher's Note}

Springer Nature remains neutral with regard to jurisdictional claims in published maps and institutional affiliations.

Received: 2 January 2019 Accepted: 18 March 2019

Published online: 16 April 2019

\section{References}

1. Perfus-Barbeoch L, Jones AM, Assmann SM (2004) Plant heterotrimeric G protein function: insights from Arabidopsis and rice mutants. Curr Opin Plant Biol 7:719-731

2. Urano D, Chen JG, Botella JR, Jones AM (2013) Heterotrimeric G protein signaling in the plant kingdom. Open Biol 3:120186

3. Apone F, Alyeshmerni N, Wiens K, Chalmers D, Chrispeels MJ, Colucci G (2003) The G-protein-coupled receptor GCR1 regulates DNA synthesis through activation of phosphatidylinositol-specific phospholipase C. Plant Physiol 133:571-579

4. Colucci G, Apone F, Alyeshmerni N, Chalmers D, Chrispeels MJ (2002) GCR1, the putative Arabidopsis G protein-coupled receptor gene is cell cycle-regulated, and its overexpression abolishes seed dormancy and shortens time to flowering. Proc Natl Acad Sci 99:4736-4741

5. Chen JG, Pandey S, Huang J, Alonso JM, Ecker JR, Assmann SM, Jones AM (2004) GCR1 can act independently of heterotrimeric G-protein in response to brassinosteroids and gibberellins in Arabidopsis seed germination. Plant Physiol 135:907-915

6. Plakidou-Dymock S, Dymock D, Hooley R (1998) A higher plant seventransmembrane receptor that influences sensitivity to cytokinins. Curr Biol 8:315-324 
7. Pandey S, Chen JG, Jones AM, Assmann SM (2006) G-protein complex mutants are hypersensitive to abscisic acid regulation of germination and postgermination development. Plant Physiol 141:243-256

8. Pandey S, Vijayakumar A (2018) Emerging themes in heterotrimeric G-protein signaling in plants. Plant Sci 270:292-300

9. Bhardwaj D, Lakhanpaul S, Tuteja N (2013) Can G-proteins be the key proteins for overcoming environmental stresses and increasing crop yield in plants? In: Tuteja N, Gill SS (eds) Plant acclimation to environmental stress. Springer, New York, pp 461-482

10. Chakravorty D, Assmann SM (2018) G protein subunit phosphorylation as a regulatory mechanism in heterotrimeric $\mathrm{G}$ protein signaling in mammals, yeast, and plants. Biochem J 475:3331-3357

11. Chakraborty N, Sharma P, Kanyuka K, Pathak RR, Choudhury D, Hooley RA, Raghuram N (2015) Transcriptome analysis of Arabidopsis GCR1 mutant reveals its roles in stress, hormones, secondary metabolism and phosphate starvation. PLoS ONE 10:e0117819

12. Chakraborty N, Sharma P, Kanyuka K, Pathak RR, Choudhury D, Hooley R, Raghuram N (2015) G-protein a-subunit (GPA1) regulates stress, nitrate and phosphate response, flavonoid biosynthesis, fruit/seed development and substantially shares GCR1 regulation in A. thaliana. Plant Mol Biol 89:559-576

13. Cone K (1989) Yet another rapid plant DNA prep. Maize Genet Coop Newslett 63:68

14. Livak KJ, Schmittgen TD (2001) Analysis of relative gene expression data using real-time quantitative PCR and the $2^{- \text {ddCT }}$ method. Methods 25:402-408

15. Park SY, Choi SR, Lim SH, Yeo YS, Kweon SJ, Bae YS, Kim KW, Im KH, Ahn SK, Ha SH, Park SU, Kim JK (2014) Identification and quantification of carotenoids in paprika fruits and cabbage, kale, and lettuce leaves. J Korean Soc Appl Biol Chem 57:355-358

16. Wellburn AR (1994) The spectral determination of chlorophylls $a$ and $b$, as well as total carotenoids, using various solvents with spectrophotometers of different resolution. J Plant Physiol 144:307-313

17. Kim TJ, Lee KB, Baek SA, Choi JH, Ha SH, Lim SH, Park SY, Yeo YS, Park SU, Kim JK (2015) Determination of lipophilic metabolites for species discrimination and quality assessment of nine leafy vegetables. J Korean Soc Appl Biol Chem 58:909-918

18. Baek SA, Jung YH, Lim SH, Park SU, Kim JK (2016) Metabolic profiling in Chinese cabbage (Brassica rapa L. subsp. pekinensis) cultivars reveals that glucosinolate content is correlated with carotenoid content. J Agric Food Chem 64:4426-4434

19. Community European (1990) Oil seeds_-determination of glucosinolates by high-performance liquid chromatography. Off J Eur Communities L170:27-34
20. Kim JK, Choi SR, Lee JY, Park SY, Song SY, Na JH, Kim SW, Kim SJ, Nou IS, Lee YH, Park SU, Kim HR (2013) Metabolic differentiation of diamondback moth (Plutella xylostella $(\mathrm{L}$.)) resistance in cabbage (Brassica oleracea L. ssp. capitata). J Agric Food Chem 61:11222-11230

21. Taddese B, Upton GJG, Bailey GR, Jordan SRD, Abdulla NY, Reeves PJ, Reynolds CA (2014) Do plants contain G protein-coupled receptors? Plant Physiol 164:287-307

22. Fahey JW, Zalcmann AT, Talalay P (2001) The chemical diversity and distribution of glucosinolates and isothiocyanates among plants. Phytochemistry 56:5-51

23. Worley B, Powers R (2013) Multivariate analysis in metabolomics. Curr Metabol 1:92-107

24. Eriksson L, Byrne T, Johansson E, Trygg J, Vikström C (2013) Multi- and megavariate data analysis, 3rd edn. Umetrics Academy, Umeå, pp 74-75

25. León P, Sheen J (2003) Sugar and hormone connections. Trends Plant Sci 8:110-116

26. Weiss D, Ori N (2007) Mechanisms of cross talk between gibberellin and other hormones. Plant Physiol 144:1240-1246

27. Cheminant S, Wild M, Bouvier F, Pelletier S, Renou JP, Erhardt M, Hayes S, Terry MJ, Genschik P, Achard P (2011) DELLAs regulate chlorophyll and carotenoid biosynthesis to prevent photooxidative damage during seedling deetiolation in Arabidopsis. Plant Cell 23:1849-1860

28. Ferruz N, Tresadern G, Pineda-Lucena A, De Fabritiis G (2016) Multibody cofactor and substrate molecular recognition in the myo-inositol monophosphatase enzyme. Sci Rep 6:30275

29. Canonne J, Froidure-Nicolas S, Rivas S (2011) Phospholipases in action during plant defense signaling. Plant Signal Behav 6:13-18

30. DeFalco TA, Bender KW, Snedden WA (2009) Breaking the code: $\mathrm{Ca}^{2+}$ sensors in plant signaling. Biochem J 425:27-40

31. Alqurashi M, Gehring C, Marondedze C (2016) Changes in the Arabidopsis thaliana proteome implicate CAMP in biotic and abiotic stress responses and changes in energy metabolism. Int J Mol Sci 17:F852

32. Roger B, Papin J, Vacher P, Raoux M, Mulot A, Dubois M, Kerr-Conte J, Voy BH, Pattou F, Charpentier G, Jonas JC, Moustaïd-Moussa N, Lang J (2011) Adenylyl cyclase 8 is central to glucagon-like peptide 1 signalling and effects of chronically elevated glucose in rat and human pancreatic beta cells. Diabetologia 54:390-402

33. Stefan E, Malleshaiah MK, Breton B, Ear PH, Bachmann V, Beyermann M, Bouvier M, Michnick SW (2011) PKA regulatory subunits mediate synergy among conserved G-protein-coupled receptor cascades. Nat Commun 2:598

34. Urano D, Phan N, Jones JC, Yang J, Huang J, Grigston J, Taylor JP, Jones AM (2012) Endocytosis of the seven-transmembrane RGS1 protein activates G-protein-coupled signalling in Arabidopsis. Nat Cell Biol 14:1079-1088

\section{Submit your manuscript to a SpringerOpen ${ }^{\circ}$ journal and benefit from:}

- Convenient online submission

- Rigorous peer review

- Open access: articles freely available online

- High visibility within the field

Retaining the copyright to your article

Submit your next manuscript at springeropen.com 